\title{
L'Union européenne et les Roms : pauvreté, haine anti-Tziganes et gouvernance de la mobilité
}

\section{Nando Sigona}

Traducteur : Francesco Ragazzi et avec l'aide de Ranieri Scognamillo

\section{(2) OpenEdition \\ Journals}

\section{Édition électronique}

URL : http://journals.openedition.org/conflits/18156

DOI : $10.4000 /$ conflits. 18156

ISSN : $1777-5345$

\section{Éditeur :}

CCLS - Centre d'études sur les conflits lilberté et sécurité, L'Harmattan

\section{Édition imprimée}

Date de publication : 5 septembre 2011

Pagination : 213-222

ISBN : 978-2-296-56086-4

ISSN : $1157-996 X$

\section{Référence électronique}

Nando Sigona, «L'Union européenne et les Roms : pauvreté, haine anti-Tziganes et gouvernance de la mobilité », Cultures \& Conflits [En ligne], 81-82 | Printemps/Été 2011, mis en ligne le 05 septembre 2012, consulté le 30 mars 2021. URL : http://journals.openedition.org/conflits/18156 ; DOI : https:// doi.org/10.4000/conflits. 18156 


\section{L'Union européenne et les Roms : pauvreté, haine anti-Tziganes et gouvernance de la mobilité 1}

\section{Nando SIGONA}

Nando Sigona est Senior researcher auprès $d u$ Refugee Studies Centre et $d u$ Centre on Migration, Policy and Society, de l'Université d'Oxford.

\section{Nouveaux instruments de gouvernance et contrôle de la mobilité des Roms}

Tors d'une récente communication au Parlement et au Conseil européen, la Commission européenne (2011) est revenue sur la question des Roms, suite aux événements relatifs aux expulsions de Roms roumains par la France l'été dernier. Cette intervention était très attendue car la Commission avait jusque là évité de prendre position sur ce sujet, laissant l'initiative aux États membres ${ }^{2}$. Cette réticence était d'autant plus marquée lorsque les Roms résidaient dans les États membres les plus riches. Dans cette communication qui pose les lignes directrices d'une intervention de coordination européenne pour les stratégies nationales d'intégration des Roms, la Commission ${ }^{3}$ explique que les 10 à 12 millions de Roms qui vivent en Europe, et dont la moitié réside au sein de l'Union Européenne (UE), sont victimes de «préjugés, d'intolérance, de discriminations et d'exclusion sociale » et que ces facteurs conditionnent de nombreux aspects de leur vie quotidienne. Plus explicitement que par le passé, la communication indique la discrimination ethnique et la pauvreté comme les causes, distinctes mais liées, de cette situation. Ce diagnostic suggère l'opportunité d'une intervention cadre qui aborde spécifiquement la situation des Roms et soit complémentaire avec la législation anti-discrimination qui, selon la Commission, « ne suffit pas à elle seule à

1. Traduction française de Francesco Ragazzi, avec l'aide de Ranieri Scognamillo.

2. Guy W., "EU Initiatives on Roma: Limitations and Ways Forward", in Sigona N., Trehan N. (eds.), Romani politics in contemporary Europe: poverty, ethnic marginalization and the neoliberal order, Londres, Palgrave, 2009, pp.23-50.

3. Commission européenne, $A$ EU framework for national Roma integration strategies up to 2020, $\operatorname{COM}(2011) 173$, Bruxelles, 5 Avril 2011. 
combattre l'exclusion des Roms ${ }^{4}$ ». Cet extrait est révélateur car il reconnait l'inadéquation d'une approche, dominante au cours des dernières années, centrée uniquement sur l'anti-discrimination. Ce changement n'est pas le fruit du hasard et participe à un processus plus général de stabilisation amorcé par l'élargissement de l'UE et par la transformation d'une partie importante des Roms de migrants non communautaires en citoyens européens, libres de circuler sur le territoire européen. Avant l'élargissement, l'approche communautaire de la mobilité (vers l'ouest) et du séjour (à l'est) des Roms était légitimée par une distinction, nette mais artificielle, entre d'une part la gravité des conditions désavantageuses et discriminatoires subies par les Roms dans les pays candidats à l'Union européenne, et d'autre part les droits fondamentaux et le bien-être garanti par l'UE à tous, y compris aux Roms. Comme l'a bien montré Simhandl, cette situation se fondait, à partir des années 1990, sur l'absence des Roms occidentaux du débat politique au niveau européen 5. Les États membres pouvaient ainsi décider d'appliquer aux pays candidats des normes sévères de protection des droits de l'homme et des minorités (appelées « critères de Copenhague »), normes qui étaient pourtant violées dans les États membres. L'élargissement de l'UE a changé la donne. En effet, avec les adhésions de la Hongrie, de la République Tchèque, de la Slovaquie, de la Slovénie et de la Pologne et plus encore avec celles de la Roumanie et de la Bulgarie, la population rom au sein de l'UE a triplé, passant d'environ 2 à plus de 6 millions. De plus, l'élargissement a permis aux Roms de migrer plus librement, transformant ainsi la démographie des communautés rom dans l'UE. En revanche, les Roms non communautaires ont rencontré de plus en plus d'obstacles pour accéder à l'UE par les voies légales, à cause de la rigidité et de la sélectivité des politiques migratoires communautaires vis-à-vis des ressortissants d'États tiers, mais aussi à cause de la restriction générale du droit d'asile, plus évident encore pour les citoyens des pays candidats à l'entrée dans l'UE comme la Macédoine, le Kosovo, la Croatie, la Serbie, la Turquie, l'Albanie et le Monténégro.

D'un point du vue institutionnel, c'est à la fin de l'année 2007 que l'on s'est rendu compte de la portée de ce changement, lorsque la première « crise nomade » a éclaté en Italie (sous le gouvernement moribond de M. Prodi). Par la suite, cette crise fut institutionnalisée par le gouvernement Berlusconi par le biais de la déclaration de l'état d'urgence dans les camps de nomades en mai $2008^{6}$, une intervention qui, pour de nombreux aspects, a servi de modèle à celle de M. Sarkozy à l'été 2010. Les mesures prises par les gouvernements français et italien ont brisé en morceaux le discours soigneusement développé par les institutions européennes à propos des Roms. Elles ont montré de

4. Ibid., p.3.

5. Simhandl K., "Beyond boundaries? Comparing the construction of the political categories 'Gypsies' and 'Roma' before and after the enlargement”, in Sigona N., Trehan N. (eds.), op. cit., $72-93$

6. Sigona N., The latest public enemy: Romanian Roma in Italy, Florence, OsservAzione, 2008. 
manière catégorique à quel point la discrimination envers les Roms, provenant également d'acteurs institutionnels, ne se limite pas aux nouveaux Étas membres, mais s'enracine profondément aussi en Europe occidentale. De plus, ces épisodes ont aussi réorienté l'attention de l'opinion publique vers la situation des Roms indigènes, trop souvent absents du débat politique. Même si elle représente un pas en avant, la communication de la Commission européenne confirme certains aspects problématiques liés aux modalités d'action des institutions européennes sur la question des Roms, en particulier vis-à-vis des interventions d'intégration pour la population majoritaire (aucune action prévue), de la réflexion politique sur les causes de la situation actuelle (aucune réflexion prévue), et des attentes placées sur les acteurs institutionnels à différents niveaux pour qu'ils interviennent réellement sur la question. Il nous paraît donc utile d'apporter une réflexion sur ces thèmes.

\section{Pauvreté et haine anti-Tziganes dans une Europe néolibérale}

Les nouveaux équilibres géopolitiques qui ont redessiné la carte de l'Europe après la chute du mur de Berlin ont été accompagnés par l'émergence et le renforcement de la doctrine économique néolibérale sur tout le continent européen, mais de façon encore plus marquée dans l'UE et ses nouveaux satellites. La transformation économique et les nouvelles politiques sociales ont entraîné la marginalisation et l'appauvrissement croissants de groupes de population qui, pour différentes raisons, n'ont pas réussi a trouver une place stable, pacifique et légitime dans le nouveau système : parmi eux des millions de Roms pour qui chômage chronique et exclusion sociale sont devenus la règle ${ }^{7}$. La paupérisation des Roms en Europe centrale et orientale s'intensifie au cours des années 1990, lorsque le triomphe néolibéral atteint son sommet. A cette époque, alors que certains bénéficiaient du nouveau bien-être, le revenu des familles rom s'écroulait à la suite de la fermeture des usines d'État et de la réduction drastique de l'emploi dans les administrations publiques. Une reconnaissance importante de ce phénomène a été apportée par l'ancien président de la Banque mondiale, James Wolfensohn, et par le financier et philanthrope Georges Soros ${ }^{8}$, deux des principaux partisans de la « Décennie de l'Inclusion Sociale des Roms », qui ont affirmé :

«Les Roms ont été les grands perdants de la transition post-communiste après 1989. Ils ont souvent été les premiers à perdre leur emploi au début des années 1990 et ils ont depuis lors été sans cesse empêchés de pénétrer à nouveau le marché du travail en raison de

7. Ringold D., Orenstein M.A., Wilkens E., Roma in an Expanding Europe: Breaking the Poverty Cycle, Washington D.C., Document de la Banque Mondiale, 2003; Roma in Central and Eastern Europe: Avoiding the Dependency Trap, UNDP/ILO Regional Human Development Report, Bratislava, UNDP, 2002; Voir aussi Sigona N., Trehan N. (eds.), op. cit.

8. Wolfensohn J.D., Soros G., "Why The Roma Matter in Europe”, Rapport présenté lors de la conférence Roma in an Expanding Europe: Challenges for the Future, Budapest, 30 juin - 1 juillet 2003 . 
leurs compétences souvent inadéquates et d'une discrimination omniprésente ».

Le cas de la Hongrie, l'un des pays de l'ex-bloc socialiste le plus avancé économiquement, est emblématique : en 1985 le taux d'emploi des hommes issus de la minorité rom était quasi identique à celui du reste de la population masculine alors qu'aujourd'hui on estime qu'au moins $70 \%$ des hommes rom sont au chômage 9 . En plus des tensions structurelles causées par une transformation économique rapide, la transition post-socialiste s'est caractérisée aussi par la recherche, dans les pays orphelins de l'Union Soviétique, de nouveaux mythes fondateurs aptes à redéfinir la relation entre l'État et la nation ${ }^{10}$. Dans un tel contexte, les mouvements nationalistes ont acquis une force croissante. Parmi eux de nombreux groupes d'extrême droite raciste et xénophobe ont réussi à se faire une place toujours plus importante dans la vie politique de presque tous les pays européens. Ce phénomène a récemment été discuté par Fox et Vermeersch ${ }^{11}$. Ils ont souligné comment l'élargissement de l'UE n'a pas abouti au dépassement attendu des nationalismes, mais semble avoir revigoré les mouvements nationalistes tout en leur apportant une nouvelle définition idéologique et tactique. Au cours de cette phase, les Roms, minorité faible dépourvue d'une réelle représentation politique, sont devenus l'un des objectifs privilégiés de campagnes racistes organisées qui ont parfois conduit à des manifestations de violence affichées. Le racisme et l'intolérance envers les Roms ne concernent cependant pas uniquement certaines franges politiques extrémistes. Les sondages effectués par l'Eurobaromètre (2007, 2008) montrent à quel point les préjugés et les stéréotypes au sujet de cette minorité sont répandus en Europe. Les données nationales concernant l'Italie offrent un tableau encore plus négatif ${ }^{12}$. De l'avis de Michael Guet, chef de la division du Conseil de l'Europe qui s'occupe de la communauté rom en Europe :

"Le caractère scandaleux de ces données, qui montrent la perception extrêmement négative des Roms dans toutes les sociétés européennes, apparait clairement lorsqu'on la compare à la perception d'autres groupes minoritaires. Alors que le débat politique autour de toutes les formes d'antisémitisme et de xénophobie peut s'appuyer sur différents instruments comme les programmes éducatifs,

9. Kertesi G., "The Employment of the Roma in the End of the 20th Century", Budapest Working Papers on the Labour Market, 2005. Pour un examen de l'impact sur la scolarisation des Roms en République Tchèque de la logique néolibérale appliquée aux systèmes éducatifs voir Nekorjak, M. "Second-class Citizens? Roma exclusion in the Czech school system", Rapport présenté lors de la conférence internationale de l'Association for the Study of Nationalities (ASN), Université de Columbia, 14-16 avril 2011.

10. Brubaker R., Nationalism reframed: Nationhood and the National Question in the New Europe, Cambridge, Cambridge University Press, 1996.

11. Fox J., Vermeersh P., "Backdoor nationalism”, European Journal of Sociology, 51(2), 2010, pp. 325-357.

12. Arrigoni P., Vitale T., "Quale legalità ? Rom e gagi a confronto », Aggiornamenti Sociali, 3, 2008, pp. 183-94. 
les plaidoyers d'acteurs politiques et de la société civile, mais aussi les actions légales, la haine anti-Tziganes demeure quelque chose de presque normal, à laquelle on ne prête aucune attention. L'absence même, pendant des décennies, d'un terme permettant de décrire le ressentiment contre les Roms en est un indicateur ${ }^{13}$ ».

Ce manque d'intérêt pour les formes de persécution et de discrimination envers les Roms a une longue histoire qui se reflète aussi, par exemple, dans l'absence, jusque récemment, de recherches sur l'extermination des Roms dans l'historiographie sur l'Holocauste ${ }^{14}$. Les termes « haine anti-Tzigane » ou « romaphobie » ne sont entrés dans le langage politique européen que récemment. La résolution du Parlement européen adoptée le 28 avril 2005 représente le premier document officiel qui aborde en détail la question des formes de discrimination envers les Roms. Elle invitait la Commission européenne à intervenir « pour combattre la haine anti-Tzigane et la romaphobie » en étant conscient « de l'importance d'éliminer d'urgence les tendances persistantes et violentes au racisme et à la discrimination raciale envers les Roms » puisque « toute impunité à l'égard d'attaques racistes, de propos haineux, d'agressions physiques perpétrées par des groupes extrémistes, d'expulsions illicites et de harcèlement policier motivés par la haine anti-Tzigane et la romaphobie contribue à saper l'État de droit et la démocratie ».

\section{Des migrants devenus citoyens européens}

Après l'élargissement de l'UE et la suppression des visas, les Roms d'Europe centrale et orientale, à la merci des changements brusques imposés par l'orientation néolibérale et par l'intolérance croissante, ont cherché, plus encore que par le passé, la possibilité de conditions de vie meilleures dans l'émigration, pour la plus grande inquiétude des chancelleries d'Europe occidentale. Jusqu'aux années 1990, les principaux pays d'émigration ont été la Macédoine, la Bosnie-Herzégovine, la Yougoslavie (Serbie, Monténégro et Kosovo) et la Roumanie. Par la suite, ce sont la Roumanie, la Bulgarie et la Slovaquie. Parmi les pays d'accueil, l'Allemagne, la France et l'Italie sont historiquement les principales destinations de la migration des Roms, alors qu'au cours des dernières années des flux importants ont également touché la Grande-Bretagne, l'Autriche et l'Espagne 15. A partir des années 1990, l'éventuelle menace représentée par l'arrivée en masse des Roms a été l'une des prin-

13. Guet M., "What is anti-Gypsyism/anti-Tsiganism/Romaphobia?”, Rapport présenté lors du séminaire sur l'anti-discrimination organisé par la présidence hongroise de la Decade for Roma Inclusion, Budapest, 16 avril 2008.

14. Nicolae V., “Anti-Gypsyism. A definition”, European Grassroots Organisation, Bucarest, ERGO, 2008.

15. Matras Y., "Romani migrations in the post-conflict era: their historical and political significance”, Cambridge Review of International Affairs, 13, 2, 2000, pp. 32-50; Sobotka E., "Romani migrations in the 1990s: perspectives on dynamic, interpretation and policy", Romani Studies, 13, 2, 2003, pp. 79-121. 
cipales raisons de l'intérêt porté envers cette population par l'UE et les autres organisations européennes du secteur, principalement le Conseil de l'Europe et l'Organization for security and cooperation in Europe (OSCE) ${ }^{16}$.

Les mesures de nature purement répressive, restrictive et dissuasive, comme les accords bilatéraux pour le retour immédiat des migrants, les échanges de renseignements et la formation des forces de polices, l'application sélective des normes sur les visas et la réduction progressive de l'effectivité du droit d'asile ont caractérisé surtout la phase de pré-élargissement ${ }^{17}$. Au fur et à mesure, d'autres types de mesures, vouées à encourager la permanence des Roms dans leur pays d'origine par la protection de leurs droits et par l'amélioration de leurs conditions socio-économiques ${ }^{18}$ sont venues s'ajouter. La raison politique de ce changement d'approche est résumée par Guglielmo et Water qui affirment :

«Bien que l'UE et les autres institutions européennes se soient d'abord concentrées sur les contrôles extérieurs à la migration, le fait que l'élargissement aux pays de l'ex-bloc soviétique soit encadré en termes de «valeurs communes » a obligé les États membres à élaborer une stratégie de gouvernance de la mobilité des Roms orientée davantage vers l'intérieur et fondée sur les droits fondamentaux et la protection des minorités 19 ».

Ainsi, à l'approche de l'élargissement, il aurait été nécessaire pour l'UE d'aborder les questions relatives aux Roms dans un registre différent, centré non pas sur l'éventualité, mais sur la manière dont les Roms allaient être intégrés dans l'UE. Le résultat de ce processus n'était pas évident et la question de la migration des Roms a failli faire dérailler le processus d'élargissement pour des pays comme la Hongrie et la Slovaquie, accusés de ne pas être en mesure de protéger les droits fondamentaux des Roms et ne pas être prêts pour la liberté de circulation. Malgré ces obstacles et une série de restrictions plus ou moins temporaires à la liberté de circulation, l'élargissement de l'Union européenne a eu lieu en 2004 et en 2007 et a changé la donne de manière importante. Ainsi, alors que dans certains pays les tensions se sont atténuées, dans d'autres, comme l'Italie et la France, la liberté de circulation des Roms est devenue une question de plus en plus pressante, tout comme l'exaspération du

16. Guy W., "Romani identity and post-Communist policy”, in Guy W. (ed.), Between Past and Future: the Roma of Central and Eastern Europe, University of Hertfordshire Press, 2001, pp. 3-32 ; Guglielmo R., Waters T.W., "Migrating towards minority status: shifting European policy towards Roma”, Journal of Common Market Studies, 43, 4, 2005, pp. 776-777 ; Vermeersch P., The Romani movement, Oxford and New York, Berghanh, 2006.

17. Rigo E., Europa di confine, Rome, Meltemi, 2007.

18. L'introduction, au cours des années 1990, de nouvelles mesures de gouvernance des flux, comme les accords bilatéraux de retour et les listes de pays tiers sûrs, ont entraîné des migrations secondaires et des changements des modèles de mobilité (Sobotka E., op. cit.).

19. Guglielmo R., Waters T.W., (eds.), op. cit., p.764. 
débat politique et la propagation de sentiments romaphobes dans l'opinion publique ${ }^{20}$.

\section{L'européanisation de la question rom}

Malgré les proclamations et les déclarations de principe, à partir des années 1990 l'intérêt prioritaire des politiques de l'UE envers les Roms a été d'en contrôler et d'en limiter la migration vers l'occident.

S'il faut reconnaitre que la tutelle des minorités, un des critères fixés pour les candidats membres à l'UE par le Conseil de Copenhague de 1993, représente un progrès important vers la reconnaissance de la protection des minorités comme l'une des normes fondatrices de la démocratie, il faut aussi souligner que la relation entre « la démocratie » et « le respect et la protection des minorités » est volontairement vague et ambigüe dans le texte de Copenhague. A ce propos, Sasse affirme:

«La formule linguistique utilisée dans les critères de l'UE fait abstraction de la notion forte de "droit des minorités ». De plus elle ne mentionne pas de "minorité nationale» et ne précise pas quels types de minorités sont concernés ${ }^{21}$ ».

En outre, même s'il ne fait pas explicitement référence aux droits, le critère soulève des objections conceptuelles et empiriques légitimes concernant le type de démocratie que l'UE voulait promouvoir dans les pays candidats. Le risque d'encourager la fragmentation de la société sur une base ethnique et d'accroître les conflits sociaux et politiques semble en effet évident, comme l'ont en quelque sorte confirmé les violentes manifestations d'intolérance et de racisme qui ont eu lieu en Hongrie au cours des derniers mois. La transposition du principe de tutelle des minorités par les pays candidats a été efficacement décrite par Tesser comme une " géopolitique de la tolérance ", soulignant le caractère intéressé et l'approche par le haut de ce processus ${ }^{22}$. De plus, comme l'ont remarqué Guglielmo et Waters, la protection des minorités formulée dans les critères de Copenhague n'est valable que pour les pays candidats à l'UE 23. Avoir délégué à l'OSCE la définition du cadre de référence pour la tutelle des minorités démontre finalement la volonté de l'UE de ne pas

20. Pour le cas de l'Italie voir Sigona N., op. cit. La situation est devenue encore plus complexe car un certain nombre de pays européens d'émigration comme la République Tchèque, la Pologne, la Slovénie et la Hongrie sont devenus des pays d'accueil pour les Roms provenant d'autres pays membres comme la Roumanie et la Slovaquie.

21. Sasse G., "Minority rights in Central and Eastern Europe before and after EU enlargement", Rapport présenté au groupe de travail Ethnic mobilization in the New Europe, Brussels, 2122 avril 2006, p. 4.

22. Tesser L., "The geopolitics of tolerance. Minority rights under EU expansion in East-Central Europe", East European Politics and Societies, 17, 3, 2003, pp. 483-532.

23. Guglielmo R., Waters T.W., op. cit. 
s'engager, au début des années 1990, dans la définition d'une réglementation propre sur les minorités. Cette attitude a progressivement évolué à la suite de l'élargissement, lorsque la réalité concrète a changé et qu'il n'a plus été possible de gérer la question des Roms uniquement en termes de gouvernance de la mobilité. En effet, même si les Roms ne circulent pas, les conditions de marginalité extrême dans lesquelles se trouvent beaucoup d'entre eux représentent en soi, puisqu'il s'agit de citoyens communautaires, une raison suffisante pour justifier l'intérêt de l'UE : la migration des Roms vers les droits a été remplacée, du moins en théorie, par la migration des droits vers les Roms. Les événements survenus en Italie ont également révélé que la discrimination systématique et institutionnelle dont sont victimes les Roms ainsi que les manifestations violentes de racisme ne se produisent pas uniquement dans les pays de l'ex-bloc soviétique mais aussi en Europe occidentale (phénomène délibérément sous-estimé par la Commission européenne au cours des années précédentes). En outre, ces faits ont permis de rappeler à la Commission que, malgré la participation décennale de l'UE à la «question rom » et les nombreux projets d'aide financés par le programme PHARE, les problèmes de la grande majorité des Roms dans les nouveaux pays membres n'ont pas été résolus, poussant ainsi bon nombre d'entre eux à migrer vers l'Ouest à la recherche d'une vie meilleure. D'après le sociologue Guy:

«Les conséquences de l'élargissement de l'UE et de l'exclusion des Roms se sont mélangées, créant une menace non seulement pour les relations entre deux États membres mais aussi pour le droit fondamental à la liberté de circulation à l'intérieur de l'UE. En décembre 2007, le Conseil européen a abordé pour la première fois la question 'de la situation très particulière des Roms de l'Union' et a invité les États membres à « user de tous les moyens pour améliorer leur inclusion (Conseil européen 2008) 24 ».

En janvier 2008, le Parlement européen a lancé un appel urgent plaidant pour l'élaboration d'une Stratégie-cadre européenne pour l'inclusion des Roms ${ }^{25}$. Un appel semblable a été lancé par les pays engagés dans la "Décennie pour l'Inclusion des Roms » et par la European Roma Policy Coalition, un réseau qui réunit les principales organisations internationales non gouvernementales qui se battent pour les droits des Roms en Europe. Après quelques réticences, ces pressions ont abouti à la communication d'avril dernier.

24. Guy W., op. cit., p. 25

25. Parlement européen, Resolution on a European strategy on the Roma, P6_TA(2008)0035, 2008, p.6. 


\section{Conclusions}

La pauvreté, l'exclusion sociale et le racisme sont trois phénomènes omniprésents dans la vie quotidienne des Roms européens qui déterminent leurs attentes et leurs espoirs pour le futur. La pauvreté et la haine anti-Tzigane sont deux phénomènes distincts, mais étroitement liés. En effet, les racines du processus d'appauvrissement de la minorité rom en Europe centrale et orientale ne peuvent se résumer au produit de politiques racistes, mais doivent plutôt être recherchées dans des facteurs systémiques comme la transformation néolibérale des économies des pays appartenant à l'ex-bloc socialiste et de l'État providence. Les réformes néolibérales de l'économie et la réduction de l'État providence ont aussi eu un impact sur les pays d'Europe occidentale où, malgré l'amnésie de l'Union européenne et de ses membres les plus riches, les conditions socio-économiques de la communauté rom sont généralement pires que celles du reste de la population. Réduire la question à un simple phénomène de discrimination raciale, comme c'est souvent le cas, limite les possibilités d'intervention et de transformation car les causes structurelles de la pauvreté des Roms et, comme l'a récemment fait remarquer Van Baar, les responsabilités des institutions européennes et nationales, ne sont ainsi pas prises en compte, comme l'a enfin reconnu la Commission européenne ${ }^{26}$. La récente communication de la Commission européenne a donné un signal positif pour reconnaitre que la réglementation anti-discrimination ne suffit pas à elle seule face à la marginalisation de la majorité des Roms. D’autre part, comme nous l'avons souligné, l'incapacité des institutions européennes à réfléchir de façon critique à leurs politiques et leurs pratiques persiste. On perçoit également chez les États membres les plus riches une difficulté à abandonner the moral high ground et l'attitude paternaliste qui caractérise les relations avec les nouveaux états membres.

En retraçant l'histoire de l'européanisation de la question rom, il convient de souligner comment, surtout dans les années 1990, et malgré le discours officiel, la limitation de la mobilité des Roms a joué un rôle de premier plan dans le choix du programme et des initiatives de l'UE et de ses États membres en la matière. Les politiques des institutions européennes ont évolué seulement à l'approche de l'élargissement de l'UE en glissant progressivement de la préoccupation face au pouvoir déstabilisant des migrations rom vers l'idée de discrimination et de tutelles des droits des minorités.

Ces transformations ne s'expliquent pas par un changement idéologique soudain chez les dirigeants européens mais bien par le changement de statut de deux millions de Roms, devenus du jour au lendemain, avec l'élargissement, des citoyens communautaires plutôt que des migrants potentiels. La

26. Van Baar H., “Europe's Romaphobia: problematisation, securitization, nomadization”, Environment and Planning D. Society and Space, 29, 2011, pp. 203-212. 
mobilité des Roms a permis, ou a imposé, de déplacer l'attention sur les conditions de vie des Roms migrants en Europe occidentale mais aussi, dans une moindre mesure, de faire la lumière sur la situation des communautés rom indigènes de ces pays, négligées par les institutions européennes depuis deux décennies.

L'élite rom européenne n'a pas réussi à obtenir un rôle politique suffisant ; la participation politique a en effet été structurée et fortement influencée par les priorités imposées par le discours néolibéral et racialisant, pendant que les communautés rom de base étaient directement touchées par les effets de la pauvreté et de l'exclusion sociale. Le racisme dans le discours politique et dans les institutions isole et affaiblit les Roms et complique la création d'alliances transversales d'intérêts capables de s'opposer efficacement au maintien du statu quo ${ }^{27}$.

27. Kovats M., "The politics of Roma identity: between nationalism and destitution", Open Democracy, 2003, p. 5; Vermeersch P., The Romani movement, Oxford and New York, Berghanh, 2006 ; Sigona N., Trehan N. (eds.), op. cit. 